\title{
TEST OF THE LOWER MANTLE SLAB PENETRATION HYPOTHESIS USING BROADBAND S WAVES
}

\author{
Susan L. Beck and Thorne Lay \\ Department of Geological Sciences, University of Michigan
}

\begin{abstract}
Broadband S and ScS displacement pulses from deep-focus earthquakes in the Sea of Okhotsk, recorded at analog and digital stations in North America and Europe, show complexities produced by velocity heterogeneity in the lower mantle. The slab beneath the Sea of Okhotsk strikes $N 40^{\circ} \mathrm{E}$, while the stations used in this study range from $N 40^{\circ} \mathrm{W}$ to $N 70^{\circ} \mathrm{E}$, allowing a test of the hypothesis that multipathing in a lower mantle slab extension gives rise to the waveform complexities. Comparisons of the $\mathrm{S}$ and $\mathrm{ScS}$ displacement pulses at a given station indicate that in many cases the direct $S$ pulses have a broader, more complex waveform than the ScS pulses. We parameterized the waveform complexity by the energy temporal centroid difference $\left(\mathrm{C}_{\mathrm{S}}-\mathrm{C}_{\mathrm{ScS}}\right)$ between the $\mathrm{S}$ and $\mathrm{ScS}$ pulses. The largest $\mathrm{C}_{\mathrm{S}}-\mathrm{C}_{\mathrm{ScS}}$ anomalies are observed along the strike of the slab. There is also a correlation between the $\mathrm{C}_{\mathrm{S}}-\mathrm{C}_{\mathrm{ScS}}$ values and ScS-S travel time residuals for stations along the strike of the slab and no correlation for off-strike azimuths. However, $\mathrm{C}_{\mathrm{S}}-\mathrm{C}_{\mathrm{ScS}}$ anomalies are observed over the entire azimuth range, including azimuths to the southwestern U.S., for which the raypaths should quickly exit any deep slab structure. These data suggest that lateral velocity heterogeneities both near the source (possibly due to slab penetration) and deep in the mantle near the $S$ wave turning points contribute to the $S$ and $S c S$ complexity.
\end{abstract}

\section{Introduction}

The question of whether subducted lithospheric slabs extend below the $670 \mathrm{~km}$ discontinuity is a fundamental problem in geophysics. The principal evidence for such lower mantle slab penetration has been travel time anomaly patterns from deep events in several subduction zones [Creager and Jordan, 1984, 1986]. The importance of this issue mandates that independent procedures be used to test the slab penetration hypothesis. Silver and Chan [1986] have argued in favor of lower mantle slab penetration beneath the Sea of Okhotsk on the basis of observations of broadband, horizontally polarized $S$ and $S c S$ phases from several deep focus earthquakes. They observed broader, more complex tails on the $S$ displacement pulses than on the ScS pulses at stations along the slab strike, which they attributed to multipathing of the $S$ phases in a near-source deep slab structure. Since the $S$ waves have shallower take-off angles than the $\mathrm{ScS}$ waves, they spend more time in the slab at azimuths along the strike of the subduction zone. For the deep-focus earthquakes, the slab must extend several hundred kilometers below the $670 \mathrm{~km}$ discontinuity in order to produce significant multipathing. In an alternate interpretation of the same data, Choy and Cormier [1986] have attributed the $S$ and $S c S$ waveform differences to radial variation of frequency dependent intrinsic attenuation in the lower mantle. They proposed a model in which attenuation of low

Copyright 1986 by the American Geophysical Union.

Paper number 6L7007.

0094-8276/86/006L-7007\$03.00 frequencies is strong in the central portion of the lower mantle. The direct $S$ phases have longer paths through this region than the $\mathrm{ScS}$ signals, giving rise to the broader tails in the $\mathrm{S}$ displacement pulses. In addition to the different nature of the heterogeneity (velocity or attenuation), these alternate interpretations can be categorized on the basis of location into two end-member models of near-source versus deep mantle heterogeneity, as shown schematically in Figure 1.

The digital data used in previous studies have a very restricted azimuthal coverage. We have analyzed a greatly expanded data set of 70 pairs of transverse component S and ScS displacement pulses from deep-focus earthquakes in the Sea of Okhotsk region from stations with relatively good azimuthal coverage in order to further document the waveform behavior. We attempt to constrain the location of the associated lower mantle heterogeneity, and assess the possibility of a deep slab extension in the lower mantle beneath the source region.

\section{Data Analysis}

The locations of events and stations used in this study are shown in Figure 2 on an azimuthal equi-distance projection centered on the source region below the Sea of Okhotsk. The strike of the Kurile Islands slab (N40 $\mathrm{E}$ ) is straddled by the available North American stations, which span azimuths from $\mathrm{N} 5^{\circ} \mathrm{E}$ to $\mathrm{N} 70^{\circ} \mathrm{E}$ and range in distance from $34^{\circ}$ to $76^{\circ}$ from the source region. This geometry allows us to test for the azimuthal pattern expected from multipathing produced by a near source slab structure.

Eleven deep-focus events were selected for their simple, impulsive waveforms. All but one have depths from 370 to $600 \mathrm{~km}$. Eight of the events produced stable SH signals across North America and were recorded at World Wide Standardized Seismograph Network (WWSSN), Canadian Seismic Network (CSN), and Regional Seismic Test Network (RSTN) stations (Figure 2). Three additional events produced stable SH radiation to Europe and were recorded at stations $\mathrm{Al}$ and $\mathrm{Bl}$ of the Graefenberg (GRF) array in West Germany. We did not use data from distances greater than $76^{\circ}$ in order to avoid complications due to lowermost mantle stratification [Lay and Helmberger, 1983].

Broadband 3-component digital data such as provided by the RSTN and GRF stations are extremely useful, but such instruments still have limited distribution (Figure 2). In order to increase the azimuthal and distance coverage of the broadband data, which is critical to constraining the location of the deep mantle heterogeneity, we extracted broadband signals from short- and long-period S and ScS phases recorded at WWSSN and CSN stations. These data were digitized and rotated to obtain tangential component short- and long-period signals for both $\mathbf{S}$ and $\mathbf{S c S}$ phases for each station. Examples of the high quality SH data that can be obtained from the analog recordings are shown in Figure 3. Broadband ground displacement pulses were obtained by simultaneous frequency domain deconvolution of the short- and long-period instruments from the analog $\mathrm{S}$ and $\mathrm{ScS}$ signals. The deconvolved short- and long-period spectra were cross-band 


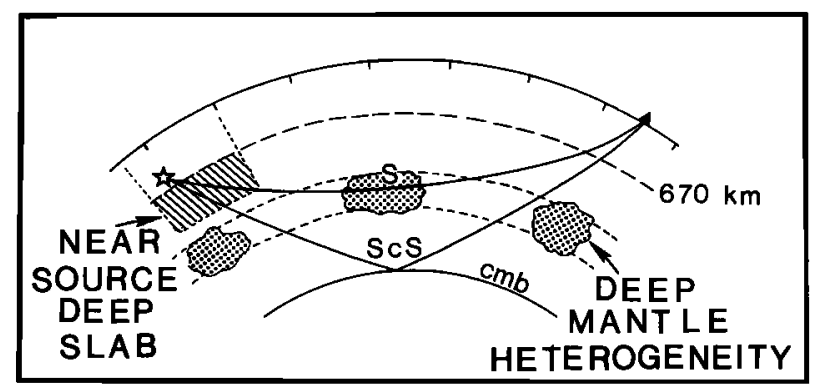

Fig. 1. A schematic view of two plausible configurations of lower mantle heterogeneity, which may explain observed waveform differences for $\mathbf{S}$ and $\mathrm{ScS}$ phases from deep focus earthquakes.

averaged in the frequency range where the instruments overlap. For most WWSSN and CSN instruments this range is between 0.15 and $0.4 \mathrm{~Hz}$. Only data with good amplitude and phase overlap in the cross-band were retained. When the resultant S and ScS displacement pulses are reconvolved with the short and longperiod instruments, the reconstituted waveforms fit the observed data quite well (Figure 4). This method enabled us to recover stable signals with a spectral bandwidth of 0.025 to $1.0 \mathrm{~Hz}$.

Comparison of the displacement pulses of S and ScS at the same station shows that the pulses usually have similar rise times, but in many cases the tail of the direct S displacement pulse is broader and more complex than the ScS pulse (Figures 4 and 5). This complexity is like that detected in the broadband data of Choy and Cormier [1986] and Silver and Chan [1986]. We have thus incorporated the data they used into this study. This includes the broadband S and ScS displacement pulses from RSTN stations for events on 4/23/84 and 2/1/84.

We have parameterized the waveform complexity by taking the difference in the $S$ and ScS energy temporal centroids, given by:

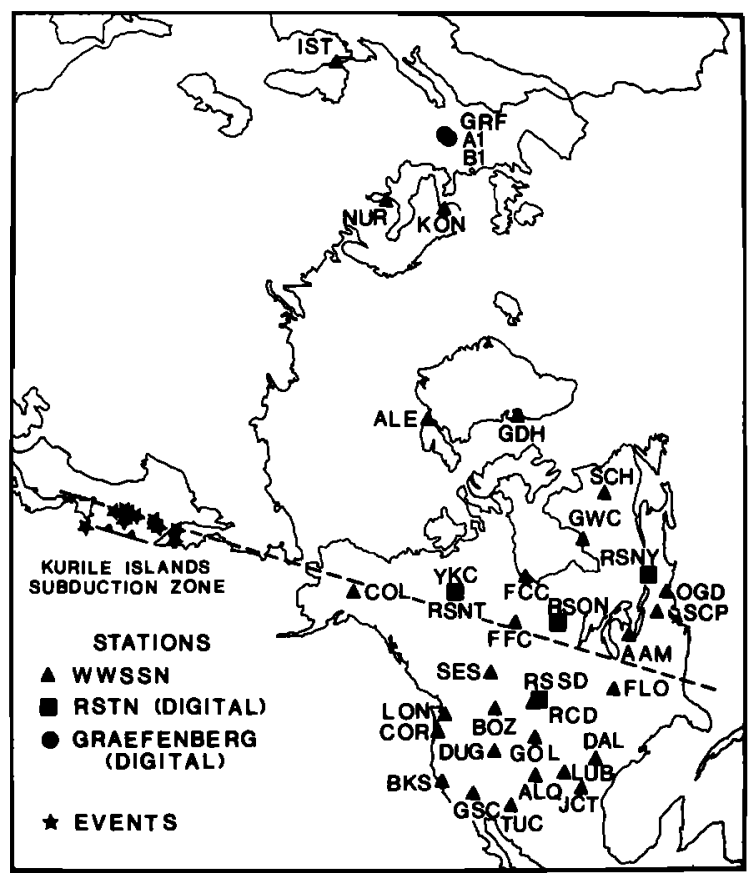

Fig. 2. An azimuthal, equi-distance projection centered on the Sea of Okhotsk source region showing the locations of events and stations used in this study.

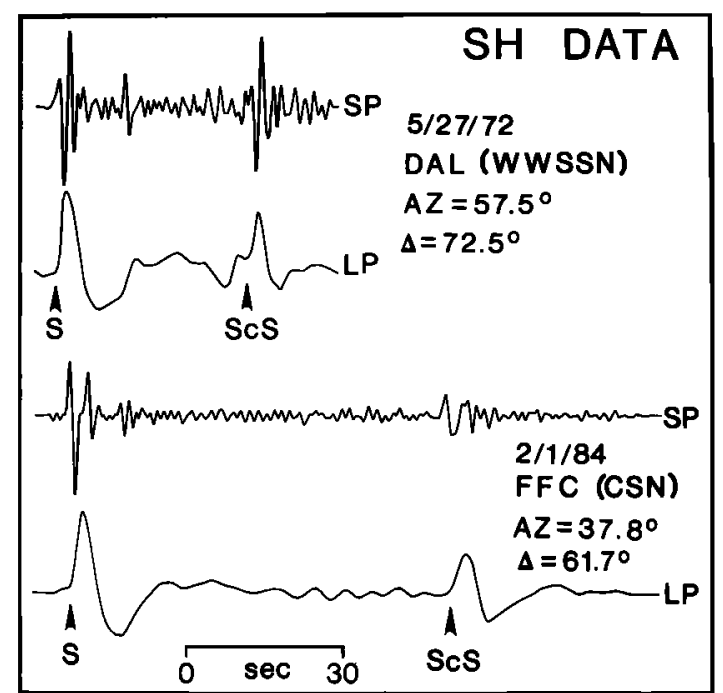

Fig. 3. Examples of the high quality short- and long-period $\mathrm{SH}$ waveforms obtained from the WWSSN and CSN stations.

$$
C_{s}-C_{S c s}=\frac{\int_{0}^{t_{m}} t S_{s}^{2}(t) d t}{\int_{0}^{t_{m}} S_{s}^{2}(t) d t}-\frac{\int_{0}^{t_{m}} t S_{S c s}^{2}(t) d t}{\int_{0}^{t_{m}} S_{S c S}^{2}(t) d t}
$$

Squaring the $\mathrm{S}$ and $\mathrm{ScS}$ displacement traces helps to account for baseline variations and any negative pulses. Comparing the $S$ and $\mathrm{ScS}$ phases for the same station suppresses receiver and source effects and allows us to combine measurements from events with

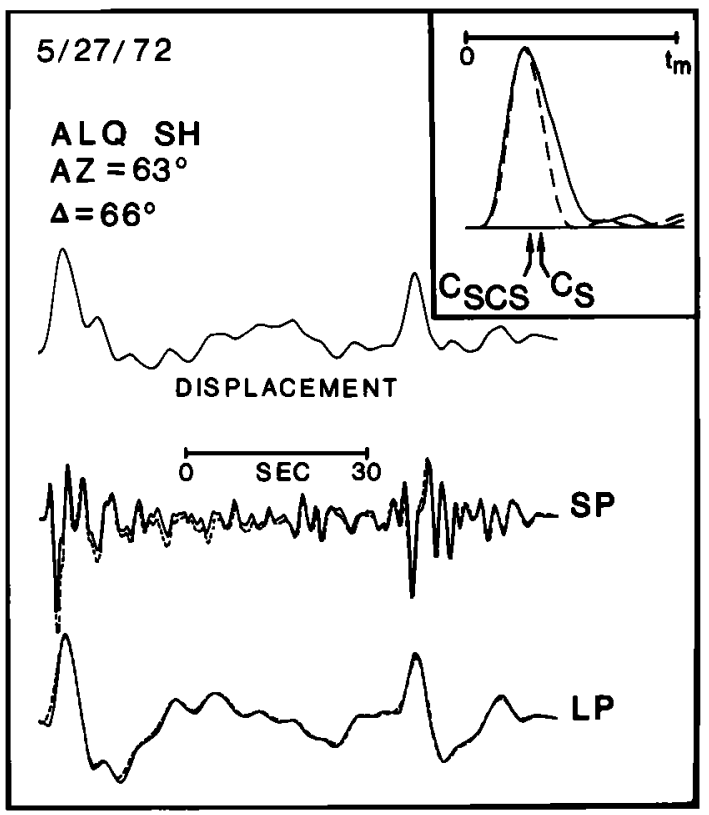

Fig. 4. An example of the simultaneous deconvolution of a broadband displacement trace from short- and long-period WWSSN signals. The dashed traces indicate the synthetic waveforms obtained by reconvolving the displacement trace with the respective instruments. The true amplitudes are preserved. The inset shows the squared S (solid line) and $\mathrm{ScS}$ (dashed line) displacement pulses and their centroids $\mathrm{C}_{\mathrm{S}}$ and $\mathrm{C}_{\mathrm{ScS}}$, computed for a signal window $t_{m}=12$ s. 


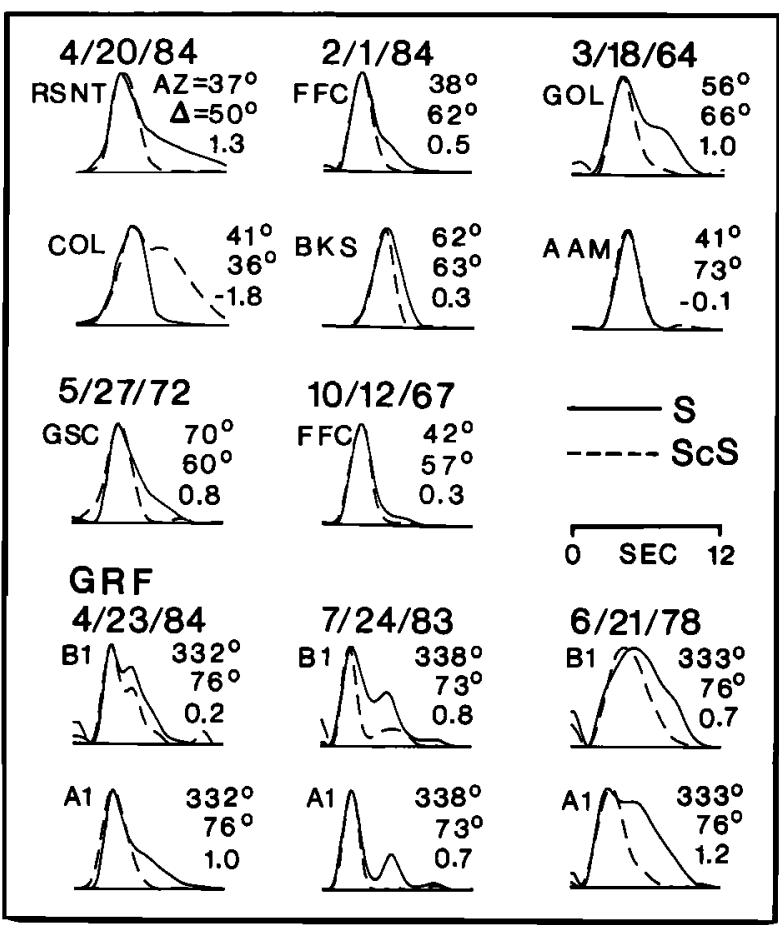

Fig. 5. Examples of the squared S (solid line) and ScS (dashed line) displacement pulses used in this study. The station azimuth, distance and centroid time difference are given for each station.

different source durations. The traces were aligned so that the rise times and peaks overlapped as in Figure 4. We used a time window $\left(\mathrm{t}_{\mathrm{m}}\right)$ of $12 \mathrm{~s}$ for the results shown in this paper, although a complete analysis using a time window of $15 \mathrm{~s}$ gave very similar results. Positive $\mathrm{C}_{\mathrm{S}}-\mathrm{C}_{\mathrm{ScS}}$ values indicate that the $\mathrm{S}$ displacement pulse is broader while negative values indicate the $\mathrm{ScS}$ pulse is broader. Examples of pairs of $\mathbf{S}$ and $\mathrm{ScS}$ squared displacement pulses are shown in Figure 5 along with the corresponding $\mathrm{C}_{\mathrm{S}}-\mathrm{C}_{\mathrm{ScS}}$ anomalies. The anomalies clearly reflect observed differences in the signals.

\section{Discussion}

Waveform complexity produced by a radially symmetric lower mantle structure should be observed at all azimuths and should have a systematic pattern with distance. For a deep slab structure we would expect to see an azimuthal pattern with respect to the slab strike. Raytracing calculations by Silver and Chan [1986] confirm that if a deep slab structure causes multipathing of the $S$ phase, stations along the strike azimuth $\left(\mathrm{N} 40^{\circ} \mathrm{E}\right)$ should have the most pronounced effects. Stations in the southwestern U.S. at azimuths out the back of the slab $\left(\mathrm{N} 50^{\circ} \mathrm{E}-\mathrm{N} 70^{\circ} \mathrm{E}\right)$, should not have significant multipathing, though it is difficult to predict three dimensional diffraction effects.

Keeping these general ideas in mind, the waveform anomalies can be inspected for the presence of any azimuthal trends. The $\mathrm{C}_{\mathrm{S}}-\mathrm{C}_{\mathrm{ScS}}$ values vary from approximately $-2 \mathrm{~s}$ to $+2 \mathrm{~s}$, with $74 \%$ of the stations giving positive values. Figure $6 \mathrm{a}, \mathrm{b}$ shows the $\mathrm{C}_{\mathrm{S}}-\mathrm{C}_{\mathrm{Scs}}$ parameter as functions of azimuth and distance. The largest anomalies, involving both the largest positive (RSNT, $\mathrm{N} 37^{\circ} \mathrm{E}$ ) and largest negative (COL, DWWSSN, N37 ${ }^{\circ} \mathrm{E}$ ) $\mathrm{C}_{\mathrm{S}}-\mathrm{C}_{\mathrm{ScS}}$ values, are observed along the strike of the slab in the Sea of Okhotsk (Figure 6a), consistent with the observations of Silver and Chan [1986]. However, the new data set also has observations along the slab strike with no waveform anomalies. There are some stations at all azimuths which show broadening of the $\mathbf{S}$ displacement pulse relative to the ScS pulse (Figures 5 and 6). Many
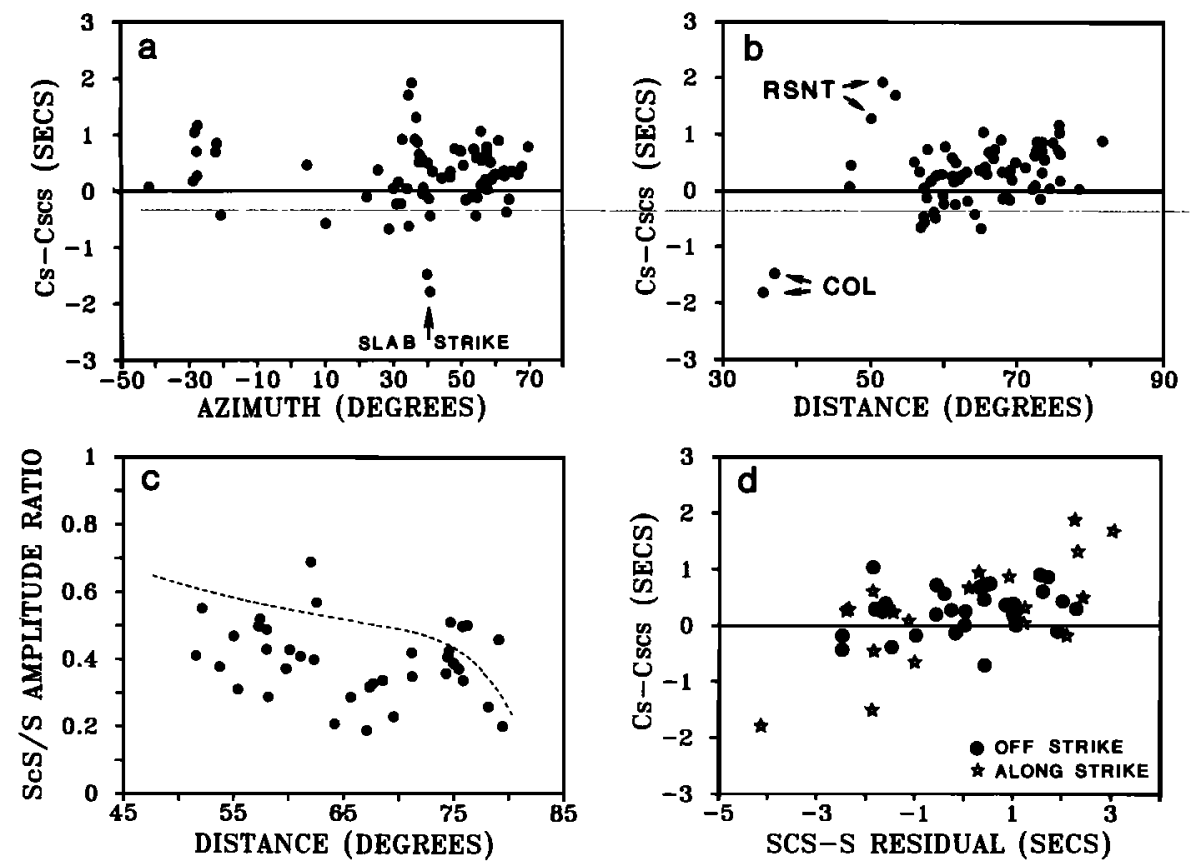

Fig. 6. Centroid differences plotted as functions of azimuth (a) and distance (b) from the Sea of Okhotsk source region. (c) Long-period peak-to-peak ScS/S amplitude ratios observed in Europe from Sea of Okhotsk events compared to the predictions for the JB model (dashed curve). (d) Centroid differences plotted versus ScS-S JB residuals with observations along the slab strike distinguished from those off the slab strike. 
stations in the southwestern U.S. at azimuths for which the raypaths should quickly exit the slab show substantial broadening of the $S$ pulses relative to the $S c S$ pulses. The $\mathrm{C}_{\mathrm{S}}-\mathrm{C}_{\mathrm{ScS}}$ anomalies also show some distance dependence (Figure $6 \mathrm{~b}$ ). The broadest ScS pulses (COL) are observed at distances of $30^{\circ}-35^{\circ}$, while the broadest $S$ pulses (RSNT) are at a distance of $50^{\circ}$. Beyond $50^{\circ}$ a relatively flat, but slightly increasing distance trend is apparent. Many of the $S$ waveforms have extra discrete arrivals (Figure 5), supporting the interpretation that the waveform differences are due to multipathing rather than attenuation. It is unlikely that a radially symmetric $Q$ model can explain the observed azimuthal and distance trends or the extra discrete shoulders in the $S$ waveforms.

Given the strong distance trends, attempts to isolate the heterogeneity using additional phases such as sS [Silver and Chan, 1986] may not be convincing. We inspected a large number of sS observations on the WWSSN recordings and observed that the sS waves are often phase shifted and significantly attenuated relative to direct $S$, making it difficult to analyze their broadband behavior.

The GRF array data in Europe (azimuth of $\mathrm{N} 30^{\circ} \mathrm{W}$ ) show broadening of the $S$ pulse relative to $\mathrm{ScS}$ for three events at both stations A1 and B1 (Figure 5). These stations are close to the downdip slab direction with a distance range of $73^{\circ}-76^{\circ}$ and therefore may be influenced by a deep slab structure. In this case, the $S$ broadening should correlate with take-off angle or distance and be most pronounced where the take-off angle of the $S$ phase is similar to the dip of the slab. Because most of the Sea of Okhotsk events do not have stable SH radiation to European azimuths, we do not have adequate distance coverage to straddle the dip direction with broadband observations. European WWSSN stations NUR and KON, at distances of $60^{\circ}$ and $64^{\circ}$ show very little, if any, broadening of the $S$ pulse for the event on 9/5/70. However, KON has a very low $\mathrm{ScS} / \mathrm{S}$ amplitude ratio. In fact, European stations in the distance range of $60^{\circ} 70^{\circ}$ consistently show long-period $\mathrm{ScS} / \mathrm{S}$ amplitude ratios (Figure $6 \mathrm{c}$ ) which are a factor of two lower than the predicted ratio for a JB earth model. A similar minimum in the ScS/S amplitude ratio from South American events was detected by Mitchell and Helmberger [1973] and attributed to a high velocity gradient above the core-mantle boundary. Further investigation is needed to establish whether defocussing by a high velocity slab extension can also produce this distance trend.

The $\mathrm{C}_{\mathrm{S}}-\mathrm{C}_{\mathrm{ScS}}$ anomalies were found to correlate with ScS-S JB travel time residuals for stations in the range $\mathrm{N} 35^{\circ} \mathrm{E}-\mathrm{N} 45^{\circ} \mathrm{E}$, which is along the slab strike (Figure $6 \mathrm{~d}$ ). The relatively broadest S pulses are associated with the largest positive ScS-S travel time residuals. This would be expected if the $S$ phase spent more time in a higher velocity slab structure. The off-strike azimuth stations in North America do not show a correlation with the ScS-S travel time residuals (Figure 6d).

The comparisons in Figure 6 reveal several fundamental aspects of the broadband $S$ wave behavior requiring several scale lengths of heterogeneities. The short scale scatter in the $\mathrm{S}$ and $\mathrm{ScS}$ waveform differences at any given azimuth or distance range is probably the result of small scale statistical heterogeneities affecting $S$ and ScS comparably. The intermediate scale azimuthal anomaly at $\mathrm{N} 40^{\circ} \mathrm{E}$ seems most easily attributed to a strong nearsource heterogeneity because of the rapid variations with ray- parameter and azimuth. It is clear that while observations along the slab strike have the most pronounced waveform differences between $\mathrm{S}$ and $\mathrm{ScS}$, these anomalies are not confined to the direct $S$ phases. ScS sometimes shows substantially greater multipathing than $S$, which is not predicted by the raytracing calculations for slab models [Silver and Chan, 1986]. The largest anomalies are isolated as functions of distance, with closer ranges having the most pronounced waveform differences. The latter aspect poses a problem in interpreting the strong correlation found between the waveform anomalies and ScS-S travel time anomalies for observations along strike because the travel time anomalies must also have a range dependence. A baseline shift toward positive anomalies is observed over the entire azimuth range. This large scale pattern indicates the presence of ubiquitous lower mantle lateral heterogeneity that preferentially affects the $S$ waves. One explanation is that the direct $S$ waves can be strongly influenced by relatively weak lateral heterogeneity near their turning points in the lower mantle, while the $\mathrm{ScS}$ reflections turn at the perfectly reflecting core-mantle boundary.

Although this expanded data set shows some evidence consistent with a near source deep mantle structure beneath the Sea of Okhotsk, the data also appear to require independent lower mantle lateral heterogeneity as well.

Acknowledgments. This research was partially supported by NSF grant EAR-8451715 and a Shell Faculty Career Initiation Grant. Acknowledgment is made to the Donors of the Petroleum Research Fund administered by the American Chemical Society, for the partial support of this research and to the Society of Exploration Geophysicists for an Educational Foundation Scholarship to S.L.B. We thank S. Schwartz, C. Lynnes and an anonymous reviewer for their helpful comments on the manuscript.

\section{References}

Choy, G. L., and V. F. Cormier, Direct measurement of the mantle attenuation operator from broadband $P$ and $S$ waveforms, J.Geophys. Res., 91, 7326-7342, 1986.

Creager, K. C. and T. H. Jordan, Slab penetration into the lower mantle, J. Geophys. Res., 89, 3031-3049, 1984.

Creager, K. C. and T. H. Jordan, Slab penetration into the lower mantle beneath the Mariana and other island arcs of the northwest Pacific, J. Geophys. Res., 91, 3573-3590, 1986.

Lay, T., and D. V. Helmberger, A lower mantle S-wave triplication and the shear velocity structure of D", Geophys. J. R. Astr. Soc., 75, 799-838, 1983.

Mitchell, B. J., and D. V. Helmberger, Shear velocities at the base of the mantle from observations of S and ScS, J. Geophys. Res. , 78, 6009-6020, 1973.

Silver, P. G., and W. W. Chan, Observations of body-wave multipathing from broad-band seismograms: Evidence for lowermantle slab penetration beneath the Sea of Okhotsk, $J$. Geophys. Res., in press, 1986.

Susan L. Beck and Thorne Lay, Dept. of Geological Sciences, University of Michigan, Ann Arbor, MI 48109.

(Received June 25, 1986; revised August 13, 1986; accepted August 15, 1986.) 